\title{
ARTICLE OPEN \\ Single injection of small-molecule amyloid accelerator results in cell death of nigral dopamine neurons in mice
}

\author{
Maria Chermenina ${ }^{1}$, Erik Chorell ${ }^{2}$, Małgorzata Pokrzywa ${ }^{3}$, Henrik Antti ${ }^{2}$, Fredrik Almqvist ${ }^{2}$, Ingrid Strömberg ${ }^{1}$ and \\ Pernilla Wittung-Stafshede ${ }^{2,4}$
}

The assembly process of a-synuclein toward amyloid fibers is linked to neurodegeneration in Parkinson's disease. In the present study, we capitalized on the in vitro discovery of a small-molecule accelerator of a-synuclein amyloid formation and assessed its effects when injected in brains of normal mice. An accelerator and an inhibitor of a-synuclein amyloid formation, as well as vehicle only, were injected into the striatum of normal mice and followed by behavioral evaluation, immunohistochemistry, and metabolomics up to six months later. The effects of molecules injected into the substantia nigra of normal and a-synuclein knockout mice were also analyzed. When accelerator or inhibitor was injected into the brain of normal mice no acute compound toxicity was found. However, 6 months after single striatal injection of accelerator, mice sensorimotor functions were impaired, whereas mice injected with inhibitor had no dysfunctions. Injection of accelerator (but not inhibitor or vehicle) into the substantia nigra revealed significant loss of tyrosine hydroxylase (TH)-positive neurons after 3 months. No loss of TH-positive neurons was found in a-synuclein knock-out mice injected with accelerator into the substantia nigra. Metabolic serum profiles from accelerator-injected normal mice matched those of newly diagnosed Parkinson's disease patients, whereas the profiles from inhibitor-injected normal mice matched controls. Single inoculation of a small-molecule amyloid accelerator may be a new approach for studies of early events during dopamine neurodegeneration in mice.

npj Parkinson's Disease (2015) 1, 15024; doi:10.1038/npjparkd.2015.24; published online 17 December 2015

\section{INTRODUCTION}

Parkinson's disease (PD) is the second most common neurological disorder and the most common movement disorder. It is characterized by widespread degeneration of subcortical structures of the brain, especially dopaminergic neurons in the substantia nigra. The assembly process of the intrinsically unstructured protein a-synuclein has been linked to the molecular basis of PD. a-Synuclein is a major component of the amyloid aggregates found in Lewy-body inclusions, which are pathological hallmarks of $\mathrm{PD}$, and mutations in a-synuclein are related to familial PD cases. $^{1,2}$ The exact function of a-synuclein is unknown, but it is suggested to be involved in synaptic vesicle release and trafficking, physiological regulation of enzymes and transporters, and participating in neuronal survival by controlling the neuronal apoptotic response ${ }^{3}$ and appears to be present in soluble and membrane-associated forms at presynaptic nerve terminals. ${ }^{4-6}$

a-Synuclein can assemble via oligomeric intermediates to amyloid fibrils and, finally, to inclusion bodies under pathological conditions. ${ }^{7}$ Although soluble a-synuclein oligomers have been proposed to be the most toxic species in PD-related neurodegeneration, ${ }^{8,9}$ recent work in animal models with preformed a-synculein fibrils have demonstrated that the amyloid fibrils themselves are toxic and can amplify in vivo, transmit to other cells, and cross the blood-brain barrier. ${ }^{10-12}$ Despite the lack of a mechanistic understanding of PD, many studies have focused on small synthetic or natural molecules that inhibit a-synuclein monomers to assemble into toxic oligomers and/or amyloid fibrils, or divert the a-synuclein assembly process toward nontoxic inert aggregates, as leads to counteract the disease. ${ }^{13,14}$ Inversely, the identification of small molecules that promote aggregation of a-synuclein into oligomers and amyloid fibers could be helpful as research tools for elucidation of early events during PD development in animals. Current animal models of PD are limited to studies of later events during disease progression as they either involve the use of toxic chemicals with non-synuclein targets that directly kill neurons or non-physiological overexpression of the a-synuclein polypeptide. ${ }^{15-18}$

FN075 is a small synthetic molecule that promotes a-synuclein amyloid formation in vitro via rapid formation of soluble oligomers. ${ }^{19}$ Recently, small-angle X-ray scattering data demonstrated that the FN075-initiated oligomers were structurally very similar to a-synuclein oligomers formed without FN075 and, as an indication of toxicity, they readily caused leakage of lipid vesicles in vitro. ${ }^{19,20}$ FN075 has a dihydro thiazolo ring-fused 2-pyridone central fragment designed to mimic a small C-terminal peptide with an extended $\beta$-sheet conformation (Figure 1a). ${ }^{19,21,22}$ Small chemical modification to the FN075 central fragment changes the properties so the molecule becomes an inhibitor of a-synuclein aggregation. ${ }^{23}$ Over the years, we have performed several in vitro characterizations of structure-function relationships for designed peptidomimetic 2-pyridone compounds on different amyloidogenic proteins, with an emphasis on a-synculein. ${ }^{19,22-24}$

In the present study, we tested whether injection of the amyloid accelerator FN075 into mice brains would promote neuronal

\footnotetext{
${ }^{1}$ Department of Integrative Medical Biology, Umeå University, Umeå, Sweden; ${ }^{2}$ Department of Chemistry, Umeå University, Umeå, Sweden and ${ }^{3}$ Airoptic Sp. z o.o., ul. Rubież 46H, Poznań, Poland.

Correspondence: F Almqvist (Fredrik.almqvist@umu.se) or I Strömberg (Ingrid.stromberg@umu.se) or P Wittung-Stafshede (pernilla.wittung@chalmers.se)

${ }^{4}$ Current address: Department of Biology and Bioengineering, Chalmers University of Technology, Gothenburg, Sweden.
}

Received 19 August 2015; revised 14 October 2015; accepted 13 November 2015 
a<smiles></smiles>
ms382
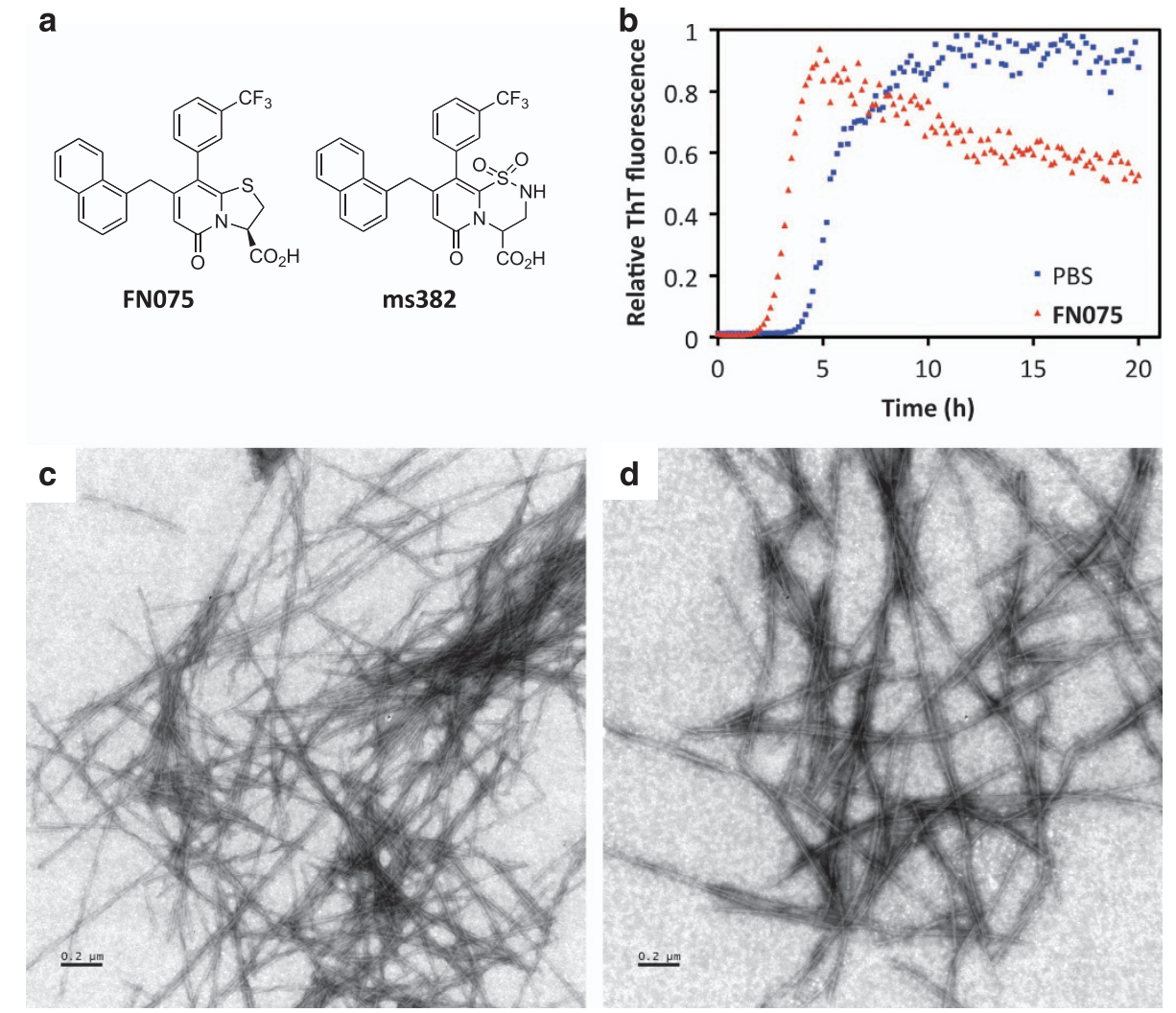

Figure 1. (a) Structures of the ring-fused 2-pyridones FN075 and ms382. (b) ThT fluorescence data for mouse $\alpha$-synculein amyloid fiber formation in the absence (blue) and presence (red) of 1:1 molar ratio of FN075. (c) EM image of $\alpha$-synculein fibers after an aggregation experiment. (d) EM image of $\alpha$-synuclein fibers formed in the presence of FN075.

damage and symptoms similar to early PD. We discovered that a single injection of FN075 into normal mice, months later, resulted in loss of dopoaminergic neurons, behavioral dysfunction, and metabolic serum profiles that paralleled those of newly diagnosed PD patients. None of these effects were found upon injection of a a-synuclein amyloid inhibitor molecule (Figure 1a), or when FN075 was injected into a-synuclein knock-out (Snca KO) mice.

\section{RESULTS}

FN075 accelerates amyloid fiber formation in vitro

FN075 accelerates formation of amyloid fibers of human a-synuclein via rapid triggering of soluble a-synculein oligomers that then readily progress to fibers at aggregation-promoting conditions. $^{19,20}$ To assure the same effects of FN075 on mouse a-synuclein, which has seven changes in its sequence compared with the human a-synuclein sequence, we expressed and purified recombinant mouse a-synuclein and tested the effect of FN075 on its amyloid fiber formation reaction using an in vitro aggregation assay based on Thioflavin T fluorescence. As expected, FN075 also accelerated amyloid fiber formation by mouse a-synuclein in vitro (Figure 1b). The resulting amyloid fibers formed in the presence of FN075 appear similar to those formed without FN075 according to electron microscopy (EM) data (Figures $1 \mathrm{c}$ and d).

Brain targets for small-molecule injection

To determine where to inject small molecules, we measured a-synuclein protein levels in different brain regions of normal mice. The levels of a-synuclein were significantly higher in the striatum as compared with the cerebellum $(P<0.0001$; Bonferroni post hoc test). The age of the mice did not influence the a-synuclein levels $\left(F_{3,28}=0.162, P>0.05\right.$; two-way analysis of variance (ANOVA); Supplementary Figure S1). Because of the high levels of a-synuclein, the striatum was selected as primary target for injection of FN075, ms382, or vehicle. For comparison, molecules were injected into the substantia nigra of normal and a-synuclein-lacking mice to study direct effects on the nigral neurons and the role of a-synuclein.

\section{Sensorimotor dysfunction}

Early behavioral impairment can be detected with the adhesive removal test, which has high sensitivity to sensorimotor dysfunction, ${ }^{25}$ detecting effects not observed by the rotarod and open-field tests. ${ }^{26}$ The basis for the adhesive removal test is the fact that in response to adhesive stimulus on the nose, normal mice raise both forelimbs toward their snout and quickly remove the stimulus with both forepaws. The sticker was removed if the animal did not swipe it off within $60 \mathrm{~s}$. The time to react to the stimulus contact was significantly different between animals given the different treatments $\left(F_{2,27}=5.917, P<0.01\right.$; one-way ANOVA). Animals given the accelerator were significantly slower $(37.2 \pm 4.7 \mathrm{~s})$ to react to the contact stimulus compared with control mice $(18.5 \pm 3.7 \mathrm{~s} ; P<0.01$; Bonferroni post hoc) and mice injected with the inhibitor $(22.4 \pm 3.7 \mathrm{~s} ; P<0.05)$. Moreover, the time to remove the stimulus was significantly different $\left(F_{2,27}=11.896, P<0.001\right)$, where the time to remove the stimulus was significantly longer for mice injected with the accelerator $(49.2 \pm 4.9 \mathrm{~s})$ when compared with mice injected with the inhibitor $(25.6 \pm 4.7 \mathrm{~s} ; P<0.01)$ and vehicle controls $(21.0 \pm 3.7 \mathrm{~s} ; P<0.001$, Bonferroni post hoc test). In addition, the calculated stimulusremoval minus stimulus-contact time was also significantly longer for animals injected with the accelerator (12.0 $\pm 3.3 \mathrm{~s})$ compared 
a

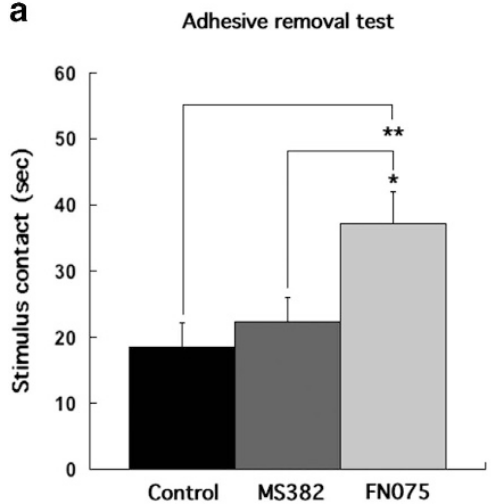

b

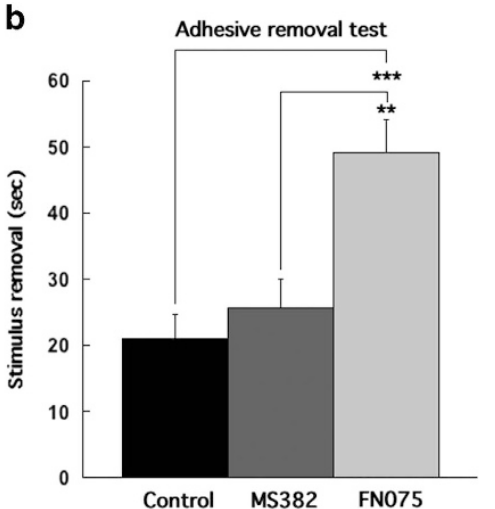

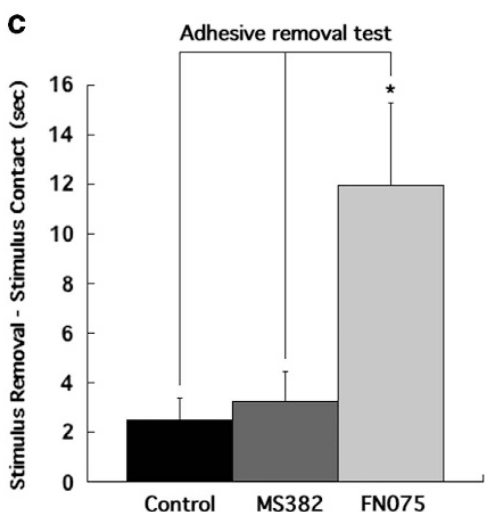

Figure 2. Data from behavioral adhesive removal test $(\mathbf{a}-\mathbf{c})$. The time to contact stimulus (a), the time to remove stimulus (b) and the time from stimulus contact to stimulus removal (c) were significantly longer in mice injected with the accelerator than the inhibitor and empty vehicle.
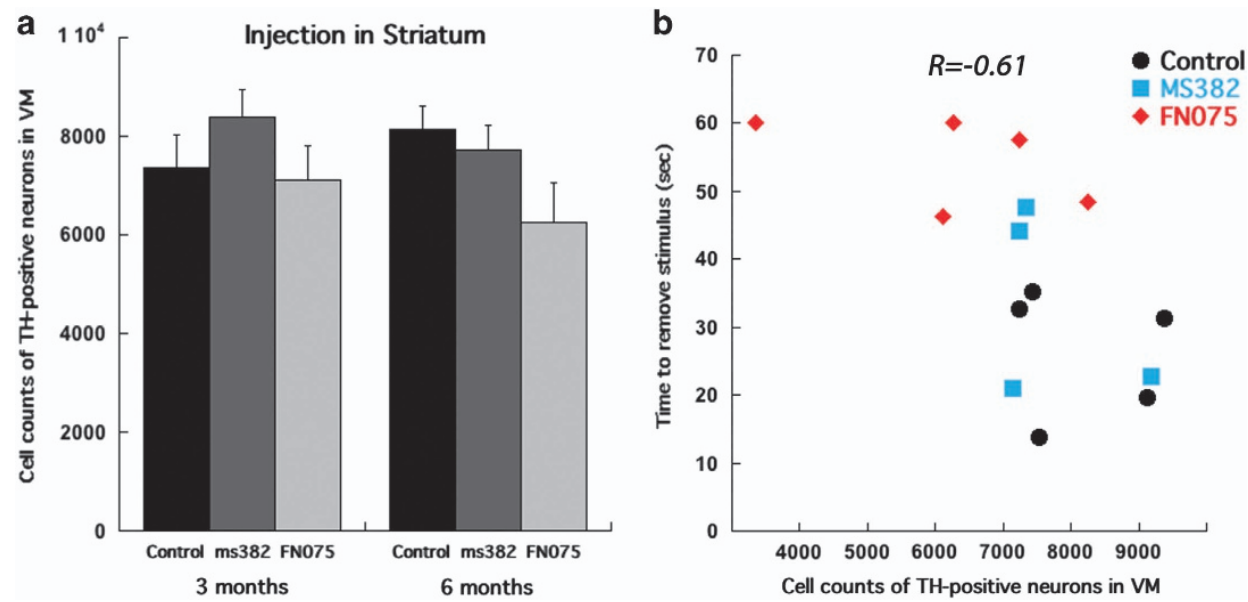

Figure 3. Cell counts of TH-positive neurons in the substantia nigra of mice injected with the accelerator, inhibitor, and vehicle into the striatum revealed (a) no significant difference between the treatments at 3 months $(n=7$ for vehicle, $n=5$ for inhibitor and $n=9$ for accelerator), but a clear decreasing trend at 6 months post-injection ( $n=5$ for vehicle, $n=4$ for inhibitor and $n=5$ for accelerator). Scatter plot demonstrates a significant negative correlation $(R=-0.61, P<0.05)$ between the time to remove the stimulus in adhesive removal test and counts of TH-positive neurons in the substantia nigra (b), ( $n=5$ for vehicle, $n=4$ for inhibitor, and $n=5$ for accelerator). ${ }^{*} P<0.05,{ }^{* *} P<0.01$, $* * * P<0.001$

with inhibitor $(3.2 \pm 1.2 \mathrm{~s} ; \quad P<0.05)$ and controls $(2.5 \pm 0.9 \mathrm{~s}$; $P<0.05$; one-way ANOVA: $F_{2,27}=6.163, P<0.01$; Figures $2 \mathrm{a}-\mathrm{c}$ ). Movies of a typical FN075-treated mouse and a control mouse performing sticker tests are shown in Supplementary Information: Supplementary Movie S1 (FN075-treated) and Supplementary Movie S2 (control).

\section{Loss of dopamine-producing neurons}

Two time points, 3 and 6 months post small-molecule injection into the striatum were chosen to evaluate survival of tyrosine hydroxylase (TH) -positive (i.e., dopamine producing) neurons in the ipsilateral substantia nigra. The analysis revealed no significant difference in survival of $\mathrm{TH}$-immunoreactive cells between the treatments at 3 months after the injection $\left(F_{2,19}=0.759 ; P>0.05\right.$; one-way ANOVA; Figure 3a). At 6 months, a moderate reduction of TH-positive neurons was found in mice injected with FN075 as compared with control and inhibitor-injected mice $\left(F_{2,11}=2.625\right.$; $P>0.05$; one-way ANOVA; Figure 3a). The number of TH-positive neurons and the adhesive removal test result exhibited a significant correlation such that mice that performed slower in the behavioral test also possessed fewer TH-positive neurons in the substantia nigra (linear correlation coefficient $R=-0.61$, $n=14, P<0.05)$. Linear regression demonstrated a significant relationship $(F(1,12)=7.232, P<0.05$; Figure $3 b)$.

The nigral cell death was more pronounced when FN075 was injected near the substantia nigra. Cell counts of TH-positive neurons in the substantia nigra, ipsilaterally to the injection, of such mice were significantly reduced $(4,347 \pm 473$ cells) already at 3 months post injection as compared with controls $(6,889 \pm 509$ cells; $P<0.01)$ and to mice injected with inhibitor ms382 $\left(6,800 \pm 639\right.$ cells; $P<0.05$; one way ANOVA: $F_{2,11}=7.893$ $P<0.01$ ) (Figures $4 a-c$ ). To assure that the loss of TH-positive cells in the FN075-injected mice was due to neuronal cell death and not loss of $\mathrm{TH}$ expression, $\mathrm{TH}$-immunoreactive sections were also processed for NeuN, as well as stained for cresyl violet (Supplementary Figure S2), which clearly visualized loss of neurons in the substantia nigra of FN075-injected mice.

\section{Neuronal damage requires a-synuclein}

To test if the loss of TH-positive nigral neurons in normal mice injected with the accelerator depended on the presence of a-synuclein, cell counts of nigral neurons were also performed in 

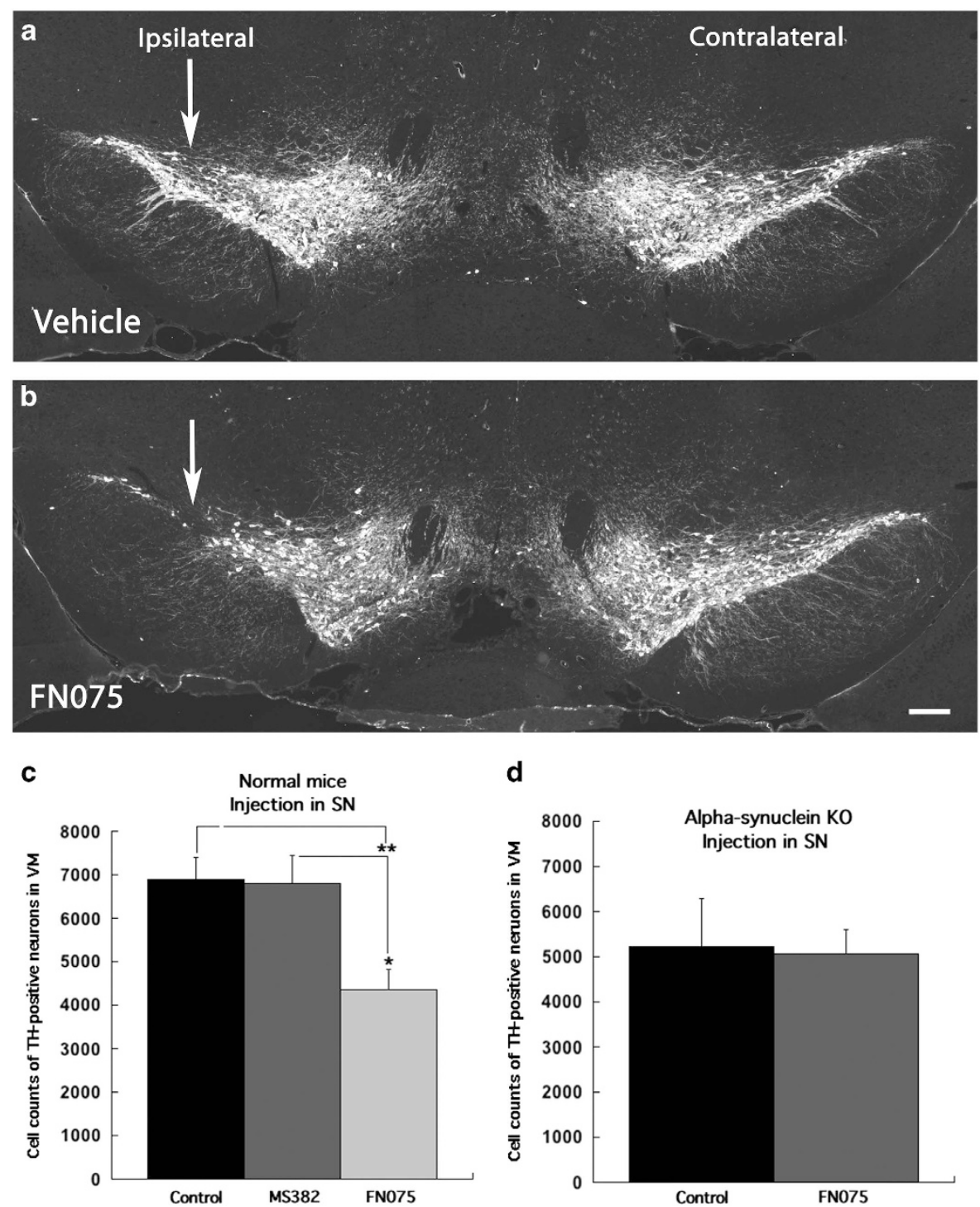

Figure 4. TH-immunohistochemistry of the substantia nigra of vehicle injection (a) and FN075 injection (b) into the substantia nigra. The depletion of TH-positive neurons is seen ipsilateral to the accelerator injection (left in b). Almost $50 \%$ reduction in counts of TH-positive neurons was found in mice injected with the accelerator compared with controls and animals treated with the inhibitor at 3 months after substantia nigra injection ( $n=6$ for vehicle, $n=4$ for inhibitor, and $n=6$ for accelerator; c). In contrast to the significant reduction of TH-positive neurons in normal mice, no differences in the number of TH-positive neurons was found in Snca KO mice after injection of FN075 near substantia nigra at 3 months postinjection ( $n=6$ for vehicle and $n=6$ for accelerator, $\mathbf{d})$. ${ }^{*} P<0.05 ;{ }^{*} P<0.01$. Scale bars: (a and $\left.\mathbf{b}\right)$ $100 \mu \mathrm{m}$.

Snca KO mice that lack a-synuclein, which had been treated with FN075 in the same way as the normal mice. The results revealed that the number of TH-positive neurons in the Snca KO mice that received injection of FN075 near the substantia nigra $(5,060 \pm 531)$ was not different from the number of neurons counted in the Snca $\mathrm{KO}$ mice that received only vehicle $\left(5,213 \pm 1078 ; t_{10}=0.128\right.$ $P>0.05$; Independent Student's $T$-test) at 3 months after the treatment (Figure 4d). This strongly implies that a-synuclein is required for FN075 to exert its effects in the normal mice, although one should be aware that there are many differences at the transcriptional level between these mice. As expected, the Snca KO mice did not show any significant differences in their adhesive removal performances due to FN075 treatment (Supplementary Figure S3).

Complementary support for the FN075 effects being mediated via a-synuclein comes from independent experiments in another in vivo model. Fruit flies overexpressing the human a-synuclein gene and fed with FN075 were found to have shorter median life span (i.e., FN075 mediates toxicity) and increased total levels of a-synuclein protein (FN075 promotes accumulation of degradation-resistant forms) as compared with vehicle-fed flies and flies fed with an inhibitor molecule (Supplementary Figures S4 and S5; Supplementary Materials). Although we did not test the effect of FN075 on normal flies, the lack of toxic effects by the inhibitor molecule in the overexpressing flies imply that the observed effects are not due to a general effect of this type of molecules.

Serum metabolomics match PD patients

Serum metabolomics data on samples taken at 1, 3, and 6 months post-injection of molecules into the striatum of the mice were analyzed for low-molecular-weight compounds (metabolites). The serum samples report on effects on homeostasis of the whole body and the underlying mechanisms relating perturbations in the nigrostriatal dopamine system to serum metabolite levels are 

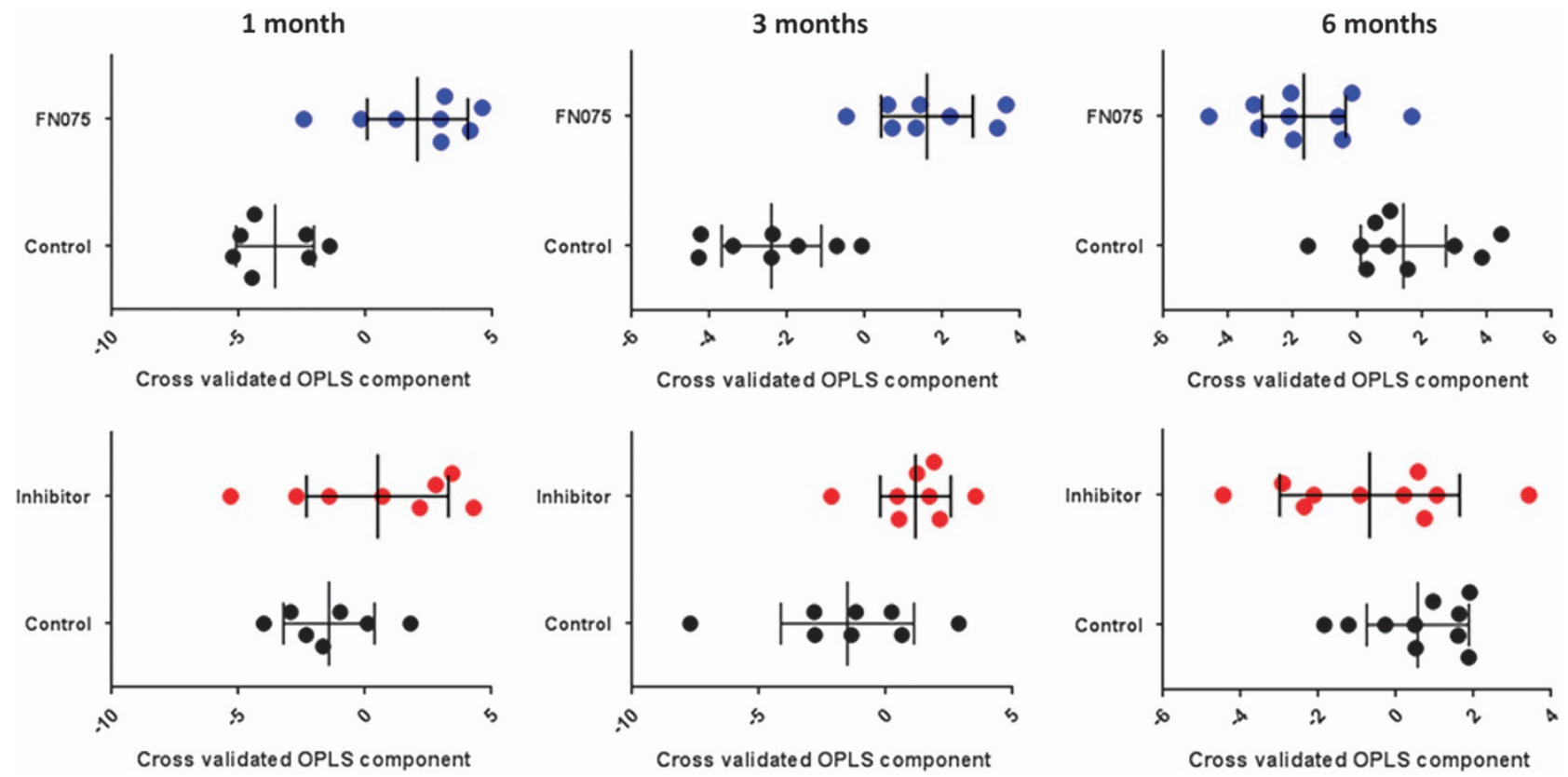

Figure 5. Data from metabolomics analysis. Upper row; Cross-validated OPLS-DA scores for pairwise OPLS-DA models between control mice (black dots) and FN075 injected mice (blue dots) $\left(P_{1 \text { month }}=0.02, P_{3 \text { months }}=0.03, P_{6 \text { months }}=0.06\right.$; based on ANOVA of cross-validated models). Lower row; Cross-validated OPLS-DA scores for pairwise OPLS-DA models between; control mice (black dots) and inhibitor-injected mice (red dots) $\left(P_{1 \text { month }}=0.23, P_{3 \text { months }}=0.17, P_{6 \text { months }}=0.71\right.$; based on ANOVA of cross validated models). Each sample class is described by the average and $95 \%$ confidence interval. In all plots each symbol represents one serum sample described by the wholemeasured metabolite profile.

not known. Multivariate analysis (Orthogonal partial least squares discriminant analysis (OPLS-DA)) revealed serum metabolite profile changes indicating that while the FN075-treated mice samples show a significant change in relation to control, such effects are not statistically significant in the inhibitor-treated mice (Figure 5). FN075-treated mice showed increases in several amino acids at the early stages post-injection (arginine, ornithine, lysine, tryptophan, serine, tyrosine, valine, glutamine, phenylalanine, asparagine, isoleucine, alanine, and pyroglutamic acid) as well as decreases in $\mathrm{C}_{15}-\mathrm{C}_{18}$ saturated and unsaturated fatty acids (including pentadecanoic acid, hexadecanoic acid, and linoleic acid) as compared with the inhibitor-treated and control mice. Six months post injection, the metabolic profile of the FN075-treated mice (compared with the controls and inhibitor treated) now displayed decreased levels of amino acids as well as increased blood sugar, hippuric acid, and cholesterol contents.

Although mechanistic interpretations are difficult, the metabolite changes at the early stages following injection for the FN075-treated mice match metabolic profiles obtained in newly diagnosed Parkinson's disease patients in terms of both identity of altered metabolites and direction of change. ${ }^{27}$ In contrast, analysis of serum from Snca KO mice demonstrated no significant difference in metabolic profile $(P=0.136)$ when comparing samples from 3-month-old FN075-injected mice with vehicleinjected mice.

\section{DISCUSSION}

In addition to finding biomarkers and treatments for neurodegenerative diseases such as Alzheimer's and Parkinson's disease, it is of high importance to also develop new research tools that allow better characterization of the molecular steps involved in the disease progression. In the present study, we investigated the consequences on the nigrostriatal dopamine system of normal mice upon a single injection of a small peptidomimetic molecule, FN075. On the basis of the in vitro observations that FN075 promotes formation of a-synuclein oligomers and amyloid fibers, ${ }^{19,23}$ we speculated that mice injected with FN075 would develop PD-like symptoms. In agreement, we discovered that FN075-injected mice had impaired sensorimotor functions, a vast loss of TH-positive neurons, and metabolic serum profiles that matched those of early-diagnosed PD patients. ${ }^{27}$ Although the molecular mechanisms behind these effects are unclear, the observations of no nigral cell death and no change in metabolic profiles for Snca KO mice injected with FN075 clearly indicate that the FN075 effects found in normal mice are dependent on a-synuclein interactions. The fruit fly data are also in agreement with FN075 targeting a-synuclein as the total a-synuclein protein levels were increased, along with reduced life span, upon FN075 feeding of the flies.

Established mouse models of PD include the use of toxic chemicals, overexpression of wild type or mutated a-synuclein, or exogenously added a-synuclein formulas. ${ }^{17,18}$ The different models all have their strengths and limitations and one has to carefully correlate the phenotypic presentations (i.e., neuronal death, Lewy bodies, ${ }^{18,28}$ motor symptoms) of the model with the research questions to be asked. For example, a-synuclein transgenic mice have failed to demonstrate loss of dopaminergic neurons, ${ }^{29-31}$ whereas overexpression of a-synuclein via viruses resulted in robust neurodegeneration along with protein aggregation. ${ }^{32,33}$ Moreover, when human wild-type a-synuclein was overexpressed under different promoters, there was divergent results concerning the presence or not of a-synuclein inclusions. ${ }^{18,34}$ However, in mice overexpressing disease-causing mutants of a-synuclein, ${ }^{35}$ inclusions resembling Lewy bodies were detected. ${ }^{36}$ Our approach differs from available animal models in that it involves a single injection of a small molecule into normal mice with the purpose of affecting endogenous a-synuclein in the brains of these mice. In some analogy to our approach, recent studies have elegantly demonstrated that injections of preformed a-synculein fibrils in animal brains cause PD pathology, and it was 
found that injected fibrils could transmit their toxic structural properties to endogenous a-synuclein polypeptides. ${ }^{10-12}$

Whereas we found neuronal cell death upon FN075-injection, none of the injected mice developed detectable amounts of a-synuclein inclusions or Lewy bodies as probed by antibodies for phosphorylated a-synuclein and ubiquitin (data not shown). This suggests that the FN075-injected mice are in early stages of the disease where no Lewy bodies have accumulated. In accord, deficits in dopaminergic neuron activity have been reported prior to neuronal loss. ${ }^{28}$ Although we cannot exclude other explanations, the molecular mechanism may involve FN075-triggered a-synuclein oligomers as the toxic species, but at concentrations too low to be detectable by conventional methods. In support, it was recently demonstrated that a-synuclein oligomers were present in neurons of PD patients prior to Lewy-body formation although a more sensitive detection method had to be developed to capture these oligomers. ${ }^{37}$ The similarity of the metabolomics data with that of PD patients strongly implies that FN075-injection promotes PD-like symptoms; this also gives indirect support for the involvement of a-synuclein. Notably, at 6 months post injection of FN075, the serum levels of several blood sugars, cholesterol and hippuric acid had increased. These compounds are all reported as potential markers of diabetes ${ }^{38-40}$ and, although more experiments are needed, may support the proposed link between type 2 diabetes and PD. ${ }^{41,42}$

\section{CONCLUSIONS}

We here evaluated the nigrostriatal dopamine system in normal mice upon single injection of a small-molecule accelerator of a-synuclein amyloid formation in vitro. Complementary experiments supported that the accelerator effects in vivo are dependent on the presence of a-synuclein. Taken together, our observations suggest that accelerator-injected mice adopt symptoms equaling early stages of PD (such as reduced sensorimotor function, loss of dopamine neurons, and altered metabolite profiles). We propose that this strategy constitutes a new approach for molecular studies of early events in neurodegeneration related to PD.

\section{MATERIALS AND METHODS}

In vitro methods

The mouse a-synuclein construct was ordered from GenScript (NJ, USA), expressed, and delivered in a pET-3a vector using the restriction sites Ndel$\mathrm{BamH}$. The construct carries the gene for mouse a-synuclein co-expressed with the 434 repressor protein which also contains an N-terminal His-tag and a caspase 7 cleavage site. Plasmid was transformed into BL21 (DE3) competent cells and grown at $37^{\circ} \mathrm{C}$ in $5 \times$ LB medium supplemented with $100 \mathrm{mg} / \mathrm{ml}$ carbenicillin and grown until $\mathrm{OD}_{600} \sim 0.6$. Protein expression was induced with $0.5 \mathrm{mmol} / \mathrm{l}$ isopropyl b-D-1-thiogalactopyranoside, and the cells were further grown overnight. The cells were centrifuged for $30 \mathrm{~min}$ at $5000 \mathrm{rpm}$, and the pellet resuspended in $8 \mathrm{~mol} / \mathrm{l}$ urea, $20 \mathrm{mmol} / \mathrm{l}$ Tris, $20 \mathrm{mmol} / \mathrm{l}$ imidazole, $\mathrm{pH} 8.0$, and sonicated on ice, followed by centrifugation at 20,000 r.p.m. for $30 \mathrm{~min}$. The supernatant was filtered and loaded on an affinity column (Ni Sepharose 6 Fast Flow, GE Healthcare, Uppsala, Sweden), equilibrated with $20 \mathrm{mmol} / \mathrm{l}$ Tris, $50 \mathrm{mmol} / \mathrm{l} \mathrm{NaCl}$, $20 \mathrm{mmol} / \mathrm{l}$ imidazole, $5 \%$ glycerol, $\mathrm{pH} 7.5$, and eluted with the same buffer but contained $250 \mathrm{mmol} / \mathrm{l}$ imidazole. For removal of the His-tag as well as co-expressed protein, peptidase caspase 7 was added in a ratio of 1:100 $(\mathrm{w} / \mathrm{w})$, together with $20 \mathrm{mmol} / \mathrm{l}$ 2-mercaptoethanol and was put to incubation over night at $4{ }^{\circ} \mathrm{C}$. Cleavage efficiency was verified with SDSpolyacrylamide gel electrophoresis, and selected fractions were diluted 1:1 $(\mathrm{v} / \mathrm{v})$ with Milli-Q water (Molsheim, France). The sample was then loaded on a anion-exchange column (HiTrap Q FF, GE Healthcare) equilibrated with $20 \mathrm{mmol} / \mathrm{l} \mathrm{Tris} \mathrm{pH} 8.0$, and eluted with a linear $\mathrm{NaCl}$ gradient of $20 \mathrm{mmol} / \mathrm{l}$ Tris, $1 \mathrm{~mol} / \mathrm{l} \mathrm{NaCl}, \mathrm{pH}$ 8.0. Finally, $a$-synuclein was run through a gel filtration column (HiLoad 16/60 Superdex 75, GE Healthcare), equilibrated with $50 \mathrm{mmol} / \mathrm{l}$ ammonium carbonate. The a-synuclein concentration was determined using the absorption at $280 \mathrm{~nm}$. a-synuclein amyloid formation assays were conducted at $37{ }^{\circ} \mathrm{C}$ with constant agitation with a 2-mm glass bead in each well $(70 \mu \mathrm{mol} / \mathrm{l}$ synuclein, $20 \mu \mathrm{mol} / \mathrm{l} \mathrm{ThT})$. Graphs are representative of at least three independent experiments. Values are reported as normalized ThT fluorescence. For transmission electron microscopy, $15-\mu \mathrm{l}$ samples from the fibrillation studies (diluted 1:10 with Milli-Q water) were applied to Formvar coated 200 mesh size copper grids. Incubated for $3 \mathrm{~min}$ and stained with $2 \%$ sodium silicon tungstate for $15 \mathrm{~s}$ before being allowed to dry. The grids were examined with a Jeol (Tokyo, Japan) model 1230 electron microscope operated at $80 \mathrm{kV}$. Images were obtained using a Gatan MultiScan 600 W camera (Abingdon, UK).

\section{Animals}

C57BI/6 (Charles River, Sulzfeld, Germany) and Snca KO (strain name: B6;129X1-Snca ${ }^{\text {tm1 Rosl }} / J$, The Jackson Laboratory, Maine, USA) female mice were used in this study. The mice were 8-week old when the study was initiated. They were housed in groups of five per cage with free access to food and water, and kept in 12- h light/dark cycle. The Northern Swedish Ethics Committee for animal experiment, in compliance with international guidelines for the protection of animals used for scientific purposes of the European Parliament and of the Council Directive (2010/63/EU), had approved all animal procedures (A 69-12 and A 24-15).

\section{Compounds to affect a-synuclein aggregation}

The 2-pyridones FN075 and ms382 (and ms400, another inhibitor used in fly studies, see Supplementary Information) were synthesized as previously described. ${ }^{21,23,43}$ FN075 is an accelerator while ms382 and ms400 are inhibitors of a-synuclein aggregation. ${ }^{19,23}$ Earlier work has shown that these compounds are monomeric in solution at the concentration used here. $^{23,24}$

\section{Intracranial injection}

The molecules were injected unilaterally either into the striatum or the substantia nigra. Mice, subjected to the striatal injection, were randomized into 3 subgroups: the first group received $2 \mu \mathrm{l} 1 \mathrm{mmol} / \mathrm{FN075}$ in 10\% dimethyl sulfoxide (DMSO) diluted in $0.9 \% \mathrm{NaCl}$ (the accelerator, $n=14$ ), animals in the second group received $2 \mu \mathrm{l} 1 \mathrm{mmol} / \mathrm{l} \mathrm{ms} 382$ in $10 \%$ DMSO diluted in $0.9 \% \mathrm{NaCl}$ (the inhibitor, $n=9$ ), and the third group received $2 \mu \mathrm{l}$ of $10 \% \mathrm{DMSO}$ in $0.9 \% \mathrm{NaCl}$ (controls, $n=12$ ). To perform nigral injections, animals were divided using the same strategy ( $n=6$ for vehicle, $n=4$ for inhibito, and $n=6$ for accelerator). Snca KO mice were randomized into two groups ( $n=6$ for vehicle and $n=6$ for accelerator) for injection into the substantia nigra. Prior to injection, the mice were anesthetized with isofluran (Baxter Medical AB, Kista, Sweden) and placed in a stereotaxic frame. A hole was drilled in the skull overlying the striatum or the substantia nigra. The coordinates for unilateral injection were: anteriorposterior (AP) $+0.12 \mathrm{~mm}$, mediolateral (ML) $-0.17 \mathrm{~mm}$, dorsoventral (DV) from the dura mater $-0.30 \mathrm{~mm}$ and $\mathrm{AP}-0.24 \mathrm{~mm}, \mathrm{ML}-0.14 \mathrm{~mm}$, DV $-0.45 \mathrm{~mm}$ for the striatum and substantia nigra, respectively. The injections were performed with a $10 \mu \mathrm{l}$ syringe $(701 \mathrm{~N}, 26 \mathrm{~s} / 2 \mathrm{2} / 3$; Hamilton Company, Nevada, USA) at the rate of $1 \mu \mathrm{l} / 2 \mathrm{~min}$. The needle was withdrawn after $2 \mathrm{~min}$ in order to avoid reflux.

\section{Behavioral analysis}

Sensorimotor functions in mice injected into the striatum 6 months postinjection or Snca KO mice injected into substantia nigra 3 mounts postinjection were tested using adhesive removal test. ${ }^{25}$ An adhesive-backed stimulus in form of a quarter of a circle, $10 \mathrm{~mm}$ in diameter, was placed on the snout of the mouse. The time to sense and the time to remove the sticker were measured with the time limit of $60 \mathrm{~s}$ and removal-contact time was calculated. The time to sense (contact) shows sensory deficits, whereas the time to remove the stimulus indicates motor impairment. ${ }^{44}$ Animal's home cage was used for the tests and all cage mates were moved temporarily to another cage. Each animal received three trials at three different days. The average time measured for these three trials were used to calculate the results.

\section{Tissue preparation and immunohistochemistry}

Transcardial perfusion using $\mathrm{Ca}^{2+}$-free Tyrode solution followed by $4 \%$ paraformaldehyde in $0.1 \mathrm{~mol} / / \mathrm{l}$ phosphate buffer $(\mathrm{pH} 7.4)$ was performed under deep sodium pentobarbital anesthesia, and thereafter the mouse brains were dissected at 3 and 6 months after the intracranial injection of 
the small molecules. The brains were postfixed in $4 \%$ paraformaldehyde overnight and rinsed in $10 \%$ sucrose in $0.1 \mathrm{~mol} / \mathrm{l}$ phosphate buffer with $0.01 \%$ sodium azide before sectioning. The brains were frozen with gaseous $\mathrm{CO}_{2}$ before being sectioned at $40 \mu \mathrm{m}$ for stereology through the entire substantia nigra, and $14 \mu \mathrm{m}$ thick sections were collected over both the striatal and substantia nigral areas for regular immunohistochemistry. The sections were rinsed in phosphate buffered saline (PBS; $0.1 \mathrm{M}, \mathrm{pH} 7.4$ ) for $15 \mathrm{~min}$ before the primary antibodies were applied. The primary antibodies used were the dopamine cell marker tyrosine hydroxylase, raised in mouse against rat tyrosine hydroxylase $(\mathrm{TH} ; 1: 1,500$; Cat \# 22941; Immunostar Inc; Hudson, WI, USA), anti-alpha Synuclein (phospho S129) raised in rabbit monoclonal antibody [EP1536Y] (1:200; Cat \# ab51253; Abcam, Cambridge, UK), anti-ubiquitin raised in rabbit polyclonal antibody (1:300; Cat \# Z0458; Dako Cytomation, Glostrup, Denmark), anti-NeuN raised in rabbit polyclonal antibody (1:500; Cat \# ab104225; Abcam, Cambrige, UK). The sections were incubated with primary antibodies diluted in $0.1 \mathrm{~mol} / \mathrm{l}$ PBS with $0.3 \%$ Triton-X-100 for $48 \mathrm{~h}$ at $4{ }^{\circ} \mathrm{C}$. After primary antibody incubations, the sections were rinsed and $5 \%$ goat serum in $0.1 \mathrm{~mol} / \mathrm{I}$ PBS was applied to avoid unspecific binding. Alexa Fluor594 (1:500, Invitrogen, Molecular probes; Eugene, OR, USA) goat anti-mouse IgG (Cat \# A11029), goat anti-rabbit IgG and Alexa Fluor488 goat anti-mouse lgG, or goat anti-rabbit lgG were used as secondary antibody. Finally, the sections were rinsed in PBS and mounted in $90 \%$ glycerol in PBS. Some sections were stained with cresyl violet to verify that neuronal death paralleled loss of TH phenotype in the nigral cells.

\section{Stereology}

To estimate the total number of $\mathrm{TH}$-positive cells in the substantia nigra ipsilateral to the injection, Optical Fractionator stereological method was used. ${ }^{45}$ Sections $(40 \mu \mathrm{m})$ were collected at an interval of 1:6 over the entire substantia nigra and processed for TH-immunohistochemistry. The total number of TH-positive neurons was counted using Stereo Investigator software (Stereolnvestigator 7, version 4.00; MicroBrightField, Inc. Vermont, USA). The substantia nigra was traced using an Olympus BX61 Microscope under low magnification using a $\times 10$ objective. TH-positive neurons were then counted under a $60 \mathrm{X}$ objective with the following stereological parameters: grid size $180 \times 180 \mu \mathrm{m}$; counting frame size $100.5 \times 75.67 \mu \mathrm{m}$; dissector height $20 \mu \mathrm{m}$. The values of the coefficient of error $(C E)<0.10$ were accepted.

\section{Western blot}

Cerebellum, striatum, and the ventral mesencephalon including the substantia nigra from C57BI/ 6 mice of 6 and 22 months of age were used to quantify the amount of a-synuclein ( $n=6$ for cerebellum, $n=12$ for striatum and $n=12$ for ventral mesencephalon). The mice were anesthetized with $4 \%$ isofluran (Baxter Medical AB, Sweden) and neck dislocated. The brains were dissected into the different brain regions and frozen in $\mathrm{CO}_{2}$. The brain tissues were sonicated (Sonifier B-12, Branson sonic power company, Danbury, CT, USA) after dilution with the lysis buffer $\left(137 \mathrm{mmol} / \mathrm{l} \mathrm{NaCl}, 2.7 \mathrm{mmol} / \mathrm{KCl}, 8.1 \mathrm{mmol} / \mathrm{l} \mathrm{Na} 2 \mathrm{HPO}_{4}, 1.5 \mathrm{mmol} / \mathrm{l} \mathrm{K \textrm {K } _ { 2 }} \mathrm{PO}_{4}\right.$, $\mathrm{pH}=7.2-7.4,1 \%$ IGEPAL CA-630, 10\% glycerol, $1 \%$ protease inhibitor). The supernatant and pellet were separated by centrifugation in 10,000 r.p.m. for $15 \mathrm{~min}$ at $4{ }^{\circ} \mathrm{C}$. a-synuclein was found in the supernatant. The BCA Protein Assay Kit (Pierce, Rockford, IL, USA) was used to quantify the protein level in the brain samples. An amount of $8 \mu \mathrm{g}$ protein from each sample was mixed 1:1 with loading buffer (5\% SDS, 10\% 2-mercaptoehtanol, $20 \%$ glycerol, $0.005 \%$ bromophenol blue, $0.125 \mathrm{~mol} / \mathrm{l}$ Tris $\mathrm{HCl}$ ) and boiled $5 \mathrm{~min}$ at $95^{\circ} \mathrm{C}$ to create a lysate to load on $12 \%$ SDS polyacrylamide gels. To determine the molecular weights of the proteins, Precision Plus Protein Standards Dual Color (Bio-Rad Laboratories AB, Solna, Sweden) was used. Transfer of proteins to a nitrocellulose membranes (Hybond ECL, Amersham Biosciences, Uppsala, Sweden) was performed using the TransBlot Semidry transfer (Bio-Rad Laboratories AB), followed by membrane blocking by $5 \%$ milk in tris buffer saline-tween (TBS-T) for $1 \mathrm{~h}$ at room temperature on a shaking table. The membranes were incubated with mouse anti-actin monoclonal antibody (1:10,000; Cat \# MAB1501R Chemicon International, Temecula, CA, USA) and rabbit anti-mouse asynuclein (1:1,000; Cat \# 2628; Cell Signaling, Danvers, MA, USA) overnight at $4{ }^{\circ} \mathrm{C}$. Washing steps $3 \times 5 \mathrm{~min}$ with TBS-T solution of the membranes were performed before the membranes were incubated with the secondary antibodies Immuno Pure IgG goat anti-mouse antibody $(1: 70,000$, Cat \# 31430; Pierce), polyclonal goat anti-rabbit immunoglobulins/HRP (1:10,000, Cat \# P0448; Dako Cytomation) for $1 \mathrm{~h}$. The membranes were rinsed in TBS-T $3 \times 5 \mathrm{~min}$ and exposed to ECL plus western blotting detection reagents for $1 \mathrm{~min}$ and were then developed (developer and fixer, 1:5, Kodak, Sigma Aldrich onto Hyperfilm ECL (GE Healthcare Life Sciences, Uppsala, Sweden). Quantification of band signal intensity was performed using NIH ImageJ $1.45 \mathrm{~s}$ program (Softonic International SA, Barcelona, Spain).

\section{Evaluation and statistics}

Throughout the experiments, all animals, slices and samples were blindcoded when analyzed. One-way ANOVA followed by Bonferroni post hoc test was used to estimate differences in behavior patterns and cell counts of TH-positive neurons in the substantia nigra. Two-way ANOVA was performed to find differences between treatments over time. The differences in level of a-synuclein protein in different brain tissue were calculated by two-way ANOVA with brain regions and time as factors followed by Bonferroni post hoc test. One-way ANOVA followed by Bonferroni post hoc test and Student's T-test was used to reveal difference in cell counts in substantia nigra injection. All statistical tests were twosided. A Person's product-moment correlation and linear regression were run to determine the relationship between the remaining number of $\mathrm{TH}$ positive neurons and the adhesive removal test result. The null hypothesis was rejected at the 0.05 level. The results are expressed as mean value \pm s.e.m. SPSS 20 statistical software (IBM Corporation, NY, USA) was used to perform all statistical tests.

\section{Metabolomic analysis}

The serum samples were extracted and derivatized according to the serum protocol for metabolomics available at Umeå Plant Science Centre (UPSC). ${ }^{46}$ Frozen 100- $\mu \mathrm{l}$ aliquots of serum, in Eppendorf tubes (Sarstedt Ref: 72.690), were first thawed in room temperature and then put on ice. Metabolite extraction was performed by addition of $900 \mu \mathrm{l}$ methanol/water extraction mix $(90: 10 \mathrm{v} / \mathrm{v}$ ) (including 11 isotopically labelled internal standards $(7 \mathrm{ng} / \mu \mathrm{l}))$ followed by rigorous shaking at $30 \mathrm{~Hz}$ for $2 \mathrm{~min}$ in a bead mill (MM 400, Retsch GmbH, Haan, Germany) and storage on ice for $120 \mathrm{~min}$ before centrifugation at 14,000 r.p.m. for $10 \mathrm{~min}$ at $4{ }^{\circ} \mathrm{C}$ (Centrifuge 5417 R, Eppendorf, Hamburg, Germany). Volume of $200 \mu \mathrm{l}$ of the supernatants were transferred to GC vials and evaporated to dryness in a speedvac (miVac, Quattro concentrator, Barnstead Genevac, Ipswitch, UK). After evaporation the samples were stored at $-80{ }^{\circ} \mathrm{C}$ until derivatization. Prior to derivatization the extracted serum samples were run for 5-10 min in the speedvac to remove possible condense. Methoxyamination, with addition of $30 \mu \mathrm{l}$ methoxyamine in pyridine $(15 \mu \mathrm{g} / \mu \mathrm{l}), 10 \mathrm{~min}$ of shaking and 60 min heating at $70^{\circ} \mathrm{C}$, was carried out with a $16 \mathrm{~h}$ reaction time (in room temperature). Trimethylsilylation, with addition of $30 \mu \mathrm{l}$ MSFTA ( $N$-methyl- $N$-trimethylsilyl-trifluoroacetamide)+1\% Trimethylchlorosilane, was carried out for $1 \mathrm{~h}$ (in room temperature). Finally, $30 \mu$ l heptane including methyl stearate $(15 \mathrm{ng} / \mu \mathrm{l})$ was added as injection standard.

\section{GC(GC)/TOF-MS analysis}

The extracted and derivatized serum samples were analysed either by $\mathrm{GC} /$ or GCGC/TOFMS. For GC/TOFMS was $1 \mu \mathrm{l}$ sample was injected splitless using an Agilent 7683 series autosampler (Agilent, Atlanta, GA, USA) into an Agilent $6980 \mathrm{GC}$ with a $10 \mathrm{~m} \times 0.18 \mathrm{~mm}$ i.d. fused-silica capillary column chemically bonded with $0.18 \mu \mathrm{m}$ DB5-MS stationary phase (J\&W Scientific, Folsom, CA, USA). The injector temperature was set at $270^{\circ} \mathrm{C}$. Helium was used as carrier gas at $(1 \mathrm{ml} / \mathrm{min})$. Purge time was set to $60 \mathrm{~s}$ at a purge flow rate of $20 \mathrm{ml} / \mathrm{min}$ and an equilibration time of $1 \mathrm{~min}$ for every analysis. Initially, the column temperature was kept at $70^{\circ} \mathrm{C}$ for 2 min and then increased to $320^{\circ} \mathrm{C}$ at $30^{\circ} \mathrm{C} / \mathrm{min}$ for $2 \mathrm{~min}$. The transfer line temperature was set at $250^{\circ} \mathrm{C}$ and the ion source temperature at $200^{\circ} \mathrm{C}$. Ions were generated by a $70-\mathrm{eV}$ electron beam at a current of $2.0 \mathrm{~mA}$. Masses were acquired from m/z 50 to 800 at a rate of $30 \mathrm{spectra} / \mathrm{s}$, and the acceleration voltage was turned on after a solvent delay of $165 \mathrm{~s}$. For GCGC/TOFMS split less injection of $1 \mu \mathrm{l}$ sample was performed using an Agilent 7683B auto sampler (injection temperature of $270^{\circ} \mathrm{C}$ ) into an a Pegasus 4D (Leco, St Joseph, MI, USA) with an Agilent 6890 gas chromatograph (Agilent Technologies, Palo Alto, GA, USA), a secondary GC oven and a quad-jet thermal modulator. Purge time was set to $60 \mathrm{~s}$ with a rate of $20 \mathrm{ml} / \mathrm{min}$ and helium was used as carrier gas ( $1 \mathrm{ml} / \mathrm{min})$. The temperature program had an initial temperature of $60^{\circ} \mathrm{C}$ for $2 \mathrm{~min}$, followed by a increase of $4{ }^{\circ} \mathrm{C} / \mathrm{min}$ up to $300^{\circ} \mathrm{C}$ where the temperature was held constant for $2 \mathrm{~min}$. The secondary oven used the same temperature program but with $a+15^{\circ} \mathrm{C}$ offset. The modulation time was $5 \mathrm{~s}$ with a hot pulse time of $0.8 \mathrm{~s}$ and a 
$1.7 \mathrm{~s}$ cooling time between the stages. The MS transfer line temperature was $300{ }^{\circ} \mathrm{C}$ and the ion source $250^{\circ} \mathrm{C}$. $70 \mathrm{eV}$ electron beams were used for the ionization and masses were recorded from 50 to $550 \mathrm{~m} / \mathrm{z}$ at a rate of $100 \mathrm{spectra} / \mathrm{s}$, with detector voltage set at $1780 \mathrm{~V}$. All serum samples were run in a randomized order. Raw data was exported to Matlab (version 14B) and processed using multivariate curve resolution by means in-house developed scripts. ${ }^{47}$ Metabolites were identified by comparisons of detected spectral profiles and retention indices with available spectral libraries.

\section{Multivariate data analysis}

Processed metabolomics data were subjected to further multivariate data analysis. OPLS-DA was performed to model the systematic variation in the metabolomics data related and orthogonal to pre-defined sample classes among the mouse serum samples, here considering, control versus FN075 treated as well as control versus inhibitor (ms382) at the three different time points. Furthermore, an OPLS-DA model was calculated to investigate the difference in metabolite profiles between FN075- and vehicle-injected Snca KO mice 3 months post injection following correction for run order bias. Cross validation was used to determine the predictive ability of the models, and ANOVA based on the cross-validated OPLS-DA models was employed to calculate $P$-values for the differences between the predefined sample classes in the respective models. The OPLS-DA model loadings were used to highlight significant metabolites. Results were displayed as cross-validated predictive scores for pairwise OPLS-DA models between control and FN075 injected mice as well as between control and inhibitor injected mice, respectively. Cross-validated predictive scores provide a visualization of the model robustness and thus of the validity of the detected metabolite patterns.

\section{ACKNOWLEDGMENTS}

We thank Jörgen Åden for preparing and purifying recombinant mouse a-synuclein and Pär Jonsson for bioinformatics support and Elin Näsström and Benny Björkblom for metabolomics analysis support. We thank Helena Edlund for providing the Snca KO mice. This study was funded by the Swedish Research Council (PWS, IS, HA, FA), the Knut and Alice Wallenberg foundation (PWS, FA), the Swedish Parkinson Foundation (IS), the Erling-Persson Familiy Foundation (HA), the Swedish Foundation for Strategic Research (FA) and the Göran Gustafsson foundation (PWS, FA).

\section{CONTRIBUTIONS}

PWS, FA, and IS designed the project. MC, EC, MP, HA performed the experiments and the data analyses. PWS, FA, MC and IS wrote the manuscript.

\section{COMPETING INTERESTS}

The authors declare no conflict of interest.

\section{REFERENCES}

1. Spillantini, M. G. et al. Alpha-synuclein in Lewy bodies. Nature $\mathbf{3 8 8}$ 839-840 (1997).

2. Polymeropoulos, M. H. et al. Mutation in the alpha-synuclein gene identified in families with Parkinson's disease. Science 276, 2045-2047 (1997)

3. Dev, K. K., Hofele, K., Barbieri, S., Buchman, V. L. \& van der Putten, H. Part II: alpha-synuclein and its molecular pathophysiological role in neurodegenerative disease. Neuropharmacology 45, 14-44 (2003)

4. Maroteaux, L., Campanelli, J. T. \& Scheller, R. H. Synuclein: a neuron-specific protein localized to the nucleus and presynaptic nerve terminal. J. Neurosci. 8 2804-2815 (1988).

5. Eliezer, D., Kutluay, E., Bussell, R. Jr. \& Browne, G. Conformational properties of alphasynuclein in its free and lipid-associated states. J. Mol. Biol. 307, 1061-1073 (2001).

6. Iwai, A. et al. The precursor protein of non-A beta component of Alzheimer's disease amyloid is a presynaptic protein of the central nervous system. Neuron 14, 467-475 (1995).

7. Uversky, V. N. Neuropathology, biochemistry, and biophysics of alpha-synuclein aggregation. J. Neurochem. 103, 17-37 (2007).

8. $\mathrm{Xu}, \mathrm{J}$. et al. Dopamine-dependent neurotoxicity of alpha-synuclein: a mechanism for selective neurodegeneration in Parkinson disease. Nat. Med. 8, 600-606 (2002)

9. Gosavi, N., Lee, H. J., Lee, J. S., Patel, S. \& Lee, S. J. Golgi fragmentation occurs in the cells with prefibrillar alpha-synuclein aggregates and precedes the formation of fibrillar inclusion. J. Biol. Chem. 277, 48984-48992 (2002).
10. Peelaerts, W. et al. alpha-Synuclein strains cause distinct synucleinopathies after local and systemic administration. Nature 522, 340-344 (2015).

11. Luk, K. C. et al. Pathological alpha-synuclein transmission initiates Parkinson-like neurodegeneration in nontransgenic mice. Science 338, 949-953 (2012).

12. Paumier, K. L. et al. Intrastriatal injection of pre-formed mouse alpha-synuclein fibrils into rats triggers alpha-synuclein pathology and bilateral nigrostriatal degeneration. Neurobiol. Dis. 82, 185-199 (2015).

13. Glabe, C. G. Structural classification of toxic amyloid oligomers. J. Biol. Chem. 283, 29639-29643 (2008).

14. Braga, C. A. et al. The anti-Parkinsonian drug selegiline delays the nucleation phase of alpha-synuclein aggregation leading to the formation of nontoxic species. J. Mol. Biol. 405, 254-273 (2011).

15. Choubey, V. et al. Mutant A53T alpha-synuclein induces neuronal death by increasing mitochondrial autophagy. J. Biol. Chem. 286, 10814-10824 (2011).

16. Sterky, F. H., Lee, S., Wibom, R., Olson, L. \& Larsson, N. G. Impaired mitochondrial transport and Parkin-independent degeneration of respiratory chaindeficient dopamine neurons in vivo. Proc. Natl Acad. Sci. USA 108, 12937-12942 (2011).

17. Chesselet, M. F., Fleming, S., Mortazavi, F. \& Meurers, B. Strengths and limitations of genetic mouse models of Parkinson's disease. Parkinsonism Relat. Disord. 14 (Suppl 2), S84-S87 (2008)

18. Blesa, J. \& Przedborski, S. Parkinson's disease: animal models and dopaminergic cell vulnerability. Front. Neuroanat. 8, 155 (2014).

19. Horvath, I. et al. Mechanisms of protein oligomerization: inhibitor of functional amyloids templates alpha-synuclein fibrillation. J. Am. Chem. Soc. 134, 3439-3444 (2012).

20. Nors Perdersen, M. et al. Direct Correlation Between Ligand-Induced alphaSynuclein Oligomers and Amyloid-like Fibril Growth. Sci. Rep. 5, 10422 (2015).

21. Aberg, V. et al. Microwave-assisted decarboxylation of bicyclic 2-pyridone scaffolds and identification of Abeta-peptide aggregation inhibitors. Org. Biomol. Chem. 3, 2817-2823 (2005).

22. Cegelski, L. et al. Small-molecule inhibitors target Escherichia coli amyloid biogenesis and biofilm formation. Nat. Chem. Biol. 5, 913-919 (2009).

23. Horvath, I. et al. Modulation of alpha-synuclein fibrillization by ring-fused 2-pyridones: templation and inhibition involve oligomers with different structure. Arch Biochem. Biophys. 532, 84-90 (2013).

24. Andersson, E. K. et al. Modulation of curli assembly and pellicle biofilm formation by chemical and protein chaperones. Chem. Biol. 20, 1245-1254 (2013).

25. Fleming, S. M. et al. Early and progressive sensorimotor anomalies in mice overexpressing wild-type human alpha-synuclein. J. Neurosci. 24, 9434-9440 (2004).

26. Richfield, E. K. et al. Behavioral and neurochemical effects of wild-type and mutated human alpha-synuclein in transgenic mice. Exp. Neurol. 175 35-48 (2002).

27. Trupp, M. et al. Metabolite and peptide levels in plasma and CSF differentiating healthy controls from patients with newly diagnosed Parkinson's disease. J. Parkinsons Dis. 4, 549-560 (2014)

28. Janezic, S. et al. Deficits in dopaminergic transmission precede neuron loss and dysfunction in a new Parkinson model. Proc. Natl Acad. Sci. USA 110 E4016-E4025 (2013)

29. Lin, X. et al. Leucine-rich repeat kinase 2 regulates the progression of neuropathology induced by Parkinson's-disease-related mutant alpha-synuclein. Neuron 64, 807-827 (2009)

30. Nuber, S. et al. Neurodegeneration and motor dysfunction in a conditional model of Parkinson's disease. J. Neurosci. 28, 2471-2484 (2008).

31. Daher, J. P. et al. Conditional transgenic mice expressing C-terminally truncated human alpha-synuclein (alphaSyn119) exhibit reduced striatal dopamine without loss of nigrostriatal pathway dopaminergic neurons. Mol. Neurodegener. 4 34 (2009).

32. St Martin, J. L. et al. Dopaminergic neuron loss and up-regulation of chaperone protein $\mathrm{mRNA}$ induced by targeted over-expression of alpha-synuclein in mouse substantia nigra. J. Neurochem. 100, 1449-1457 (2007).

33. Theodore, S., Cao, S., McLean, P. J. \& Standaert, D. G. Targeted overexpression of human alpha-synuclein triggers microglial activation and an adaptive immune response in a mouse model of Parkinson disease. J. Neuropathol. Exp. Neurol. 67 1149-1158 (2008).

34. Thiruchelvam, M. J., Powers, J. M., Cory-Slechta, D. A. \& Richfield, E. K. Risk factors for dopaminergic neuron loss in human alpha-synuclein transgenic mice. Eur. J. Neurosci. 19, 845-854 (2004).

35. Zarranz, J. J. et al. The new mutation, E46K, of alpha-synuclein causes Parkinson and Lewy body dementia. Ann. Neurol. 55, 164-173 (2004).

36. Wakamatsu, M. et al. Selective loss of nigral dopamine neurons induced by overexpression of truncated human alpha-synuclein in mice. Neurobiol. Aging 29, 574-585 (2008). 
37. Roberts, R. F., Wade-Martins, R. \& Alegre-Abarrategui, J. Direct visualization of alpha-synuclein oligomers reveals previously undetected pathology in Parkinson's disease brain. Brain 138, 1642-1657 (2015).

38. Lu, L. et al. Diabetes and risk of Parkinson's disease: an updated meta-analysis of case-control studies. PLOS ONE 9, e85781 (2014).

39. Kim, J. Y. et al. Metabolic profiling of plasma in overweight/obese and lean men using ultra performance liquid chromatography and Q-TOF mass spectrometry (UPLC-Q-TOF MS). J Proteome Res 9, 4368-4375 (2010).

40. Lees, H. J., Swann, J. R., Wilson, I. D., Nicholson, J. K. \& Holmes, E. Hippurate: the natural history of a mammalian-microbial cometabolite. J. Proteome Res. 12, 1527-1546 (2013).

41. Santiago, J. A. \& Potashkin, J. A. System-based approaches to decode the molecular links in Parkinson's disease and diabetes. Neurobiol. Dis. 72 (Pt A), 84-91 (2014).

42. Sharma, S. K. et al. Insulin-degrading enzyme prevents alpha-synuclein fibril formation in a nonproteolytical manner. Sci. Rep. 5, 12531 (2015).

43. Sellstedt, M. \& Almqvist, F. A novel heterocyclic scaffold formed by ring expansion of a cyclic sulfone to sulfonamides. Org. Lett. 11, 5470-5472 (2009).
44. Schaar, K. L., Brenneman, M. M. \& Savitz, S. I. Functional assessments in the rodent stroke model. Exp. Transl. Stroke Med. 2, 13 (2010).

45. West, M. J. New stereological methods for counting neurons. Neurobiol. Aging 14, 275-285 (1993)

46. A, J. et al. Extraction and GC/MS analysis of the human blood plasma metabolome. Anal. Chem. 77, 8086-8094 (2005).

47. Jonsson, P. et al. High-throughput data analysis for detecting and identifying differences between samples in GC/MS-based metabolomic analyses. Anal. Chem. 77, 5635-5642 (2005).

\section{(c) (i)}

This work is licensed under a Creative Commons Attribution 4.0 International License. The images or other third party material in this article are included in the article's Creative Commons license, unless indicated otherwise in the credit line; if the material is not included under the Creative Commons license, users will need to obtain permission from the license holder to reproduce the material. To view a copy of this license, visit http://creativecommons.org/licenses/ by/4.0/

Supplementary Information accompanies the paper on the npj Parkinson's Disease website (http://www.nature.com/npjparkd) 